\title{
Newly Developed Modified ALBI Grade Shows Better Prognostic and Predictive Value for Hepatocellular Carcinoma
}

\author{
Masatoshi Kudo \\ Department of Gastroenterology and Hepatology, Kindai University Faculty of Medicine, Osaka-Sayama, Japan
}

\section{The Child-Pugh and Albumin-Bilirubin Grades}

Conventionally, the Child-Pugh grade is used as an index of hepatic functional reserve in patients with hepatocellular carcinoma (HCC). However, it has a few drawbacks. Two of its five constituent factors, hepatic encephalopathy and ascites, are subjective, and serum albumin levels and ascites are confounding factors [1]. Moreover, the distribution of patients across grades is heavily skewed, with Child-Pugh A patients accounting for about two-thirds of all patients (Table 1). In 2015, Johnson et al. [2] proposed the albumin-bilirubin (ALBI) grade as a new evidence-based approach. The original formula for the ALBI grade used only serum albumin and bilirubin levels. Specifically, a numeric ALBI score is first calculated using the formula $\left(\log _{10}\right.$ bilirubin $\left.[\mu \mathrm{mol} / \mathrm{L}] \times 0.66\right)$ $+($ albumin $[\mathrm{g} / \mathrm{L}] \times-0.0852)$, and then an ALBI grade of $1-3$ is assigned based on the result. A score of -2.60 or lower is grade $1,-2.60$ to -1.39 is grade 2 , and -1.39 or higher is grade 3. A Japanese cohort study showed that this ALBI grade enables more detailed prognostic classification than the Child-Pugh grade; in addition, other studies in Chinese, European, and American cohorts showed that the performance of the ALBI for stratifying liver function in these populations was reproducible. The finding that HCC patients in Japan, Europe, the USA, and China with Child-Pugh A and ALBI grade 1 had a better karger@karger.com www.karger.com/lic

Karger!

bOPEN ACCESS
C 2021 The Author(s).

Published by S. Karger AG, Basel

This is an Open Access article licensed under the Creative Commons (http://www.karger.com/Services/OpenAccessLicense), applicable to the online version of the article only. Usage and distribution for commercial purposes requires written permission. Attribution-NonCommercial-4.0 International License (CC BY-NC)

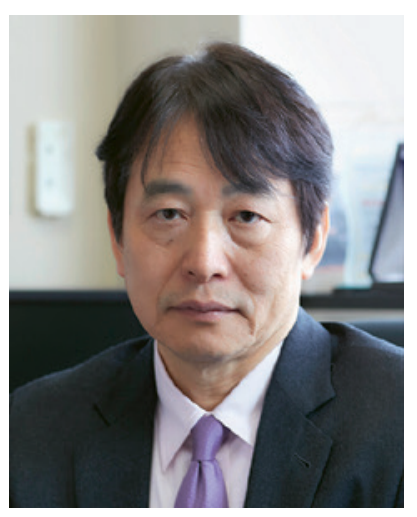

Prof. M. Kudo

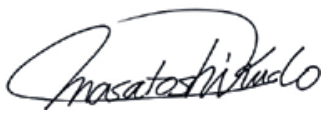

Editor Liver Cancer prognosis than those with ALBI grade 2 further demonstrated the usefulness of the ALBI grade system [2]. Therefore, the ALBI grade and ALBI score are now becoming used widely in routine clinical practice. A simpler calculation, called the EZ (easy)-ALBI score (calculated as T-Bil $[\mathrm{mg} / \mathrm{dL}]-9 \times$ Alb $[\mathrm{g} / \mathrm{dL}])$ was also proposed recently [3].

However, there are some issues with the ALBI grade. As shown in Tables 1 and 2, the distribution of HCC patients across grades is highly skewed: few patients are classified as ALBI grade 3, and ALBI grade 2 includes a very wide-ranging population (accounting for $52-65 \%$ of all HCC patients) (Table 1). This is a major problem because

Correspondence to:

Masatoshi Kudo, m-kudo@med.kindai.ac.jp 
Table 1. Frequency of Child-Pugh grade and ALBI grade

\begin{tabular}{|c|c|c|c|c|c|c|}
\hline \multirow[t]{2}{*}{ Ethnic group } & \multicolumn{3}{|c|}{ Child-Pugh grade, $n$ (\%) } & \multicolumn{3}{|c|}{ ALBI grade, $n(\%)$} \\
\hline & $A$ & B & C & 1 & 2 & 3 \\
\hline \multicolumn{7}{|l|}{ Japanese } \\
\hline $\begin{array}{l}\quad(n=2,596) \\
\text { Chinese }\end{array}$ & $1,741(67)$ & $684(36)$ & $171(7)$ & $650(25)$ & $1,699(65)$ & $247(10)$ \\
\hline $\begin{array}{l}(n=1,823) \\
\left(n=1,705^{*}\right)\end{array}$ & $1,227(67)$ & $447(25)$ & $149(8)$ & $591(35)$ & 915 (54) & $199(12)$ \\
\hline European & & & & & & \\
\hline $\begin{array}{l}\quad(n=1,108) \\
\text { USA }\end{array}$ & $728(66)$ & $318(29)$ & $62(6)$ & $324(29)$ & $643(58)$ & $141(13)$ \\
\hline $\begin{array}{l}(n=308) \\
\left(n=319^{*}\right)\end{array}$ & $175(57)$ & $96(31)$ & $37(12)$ & $82(26)$ & $167(\mathbf{5 2})$ & $70(22)$ \\
\hline
\end{tabular}

Bold values indicate importance. * Total number of patients assigned an ALBI grade. Cited from [2].

Table 2. Frequency of Child-Pugh grade and mALBI grade

\begin{tabular}{|c|c|c|c|c|c|c|c|}
\hline & \multicolumn{3}{|c|}{ Child-Pugh grade, $n$ (\%) } & \multicolumn{4}{|c|}{ mALBI grade, $n(\%)$} \\
\hline & A & B & C & 1 & $2 a$ & $2 b$ & 3 \\
\hline $\begin{array}{l}\text { LCSGJ [8] (all patients) } \\
\qquad \begin{array}{l}(n=85,636) \\
\left(n=72,551^{*}\right)\end{array}\end{array}$ & $65,570(77)$ & $16,822(20)$ & $3,244(3)$ & $35,741(39)$ & $21,266(23)$ & $29,364(32)$ & $6,180(6)$ \\
\hline $\begin{array}{l}\text { LCSGJ [8] (systemic therapy) } \\
\qquad \begin{array}{l}(n=1,103) \\
\left(n=1,161^{*}\right)\end{array}\end{array}$ & 815 (74) & $271(25)$ & $17(1)$ & $410(35)$ & $268(23)$ & $438(38)$ & $45(4)$ \\
\hline $\begin{array}{l}\text { Hiraoka et al. [4] } \\
\quad(n=6,649)\end{array}$ & $4,812(72)$ & $1,546(23)$ & $291(5)$ & $2,531(38)$ & $1,542(23)$ & $2,178(33)$ & $488(7)$ \\
\hline $\begin{array}{l}\text { Tada et al. [9] } \\
\qquad(n=524)\end{array}$ & $448(85)$ & $73(14)$ & $3(1)$ & $165(32)$ & $131(\mathbf{2 5})$ & $210(40)$ & $18(3)$ \\
\hline
\end{tabular}

Bold values indicate importance. * Total number of patients assigned a mALBI grade.

ALBI grade 2 patients who almost meet grade 1 criteria, and those who almost meet grade 3 criteria, have dramatically different hepatic functional reserves, but both are classified as grade 2 nonetheless.

\section{Modified ALBI Grade}

In 2017, Hiraoka et al. [4] undertook a detailed analysis based on the results of a nationwide survey of 46,681 patients conducted by the Liver Cancer Study Group of Japan; they proposed that ALBI grade 2 be subdivided into ALBI grades $2 \mathrm{a}$ and $2 \mathrm{~b}$ [5]. To separate the subgroups, they used a cut-off value of $30 \%$ for the indocya- nine green retention rate at $15 \mathrm{~min}$ (ICG-R15), making it a clinically relevant subclassification of the ALBI grade. They set the modified ALBI (mALBI) 2a as the borderline hepatic functional reserve for curative resection; this is because the well-known Makuuchi criteria for hepatic resection [6] state that patients with an ICG-R15 of 30\% or less are eligible for sub-segmentectomy. They calculated the cut-off ALBI score corresponding to $30 \%$ ICG-R15 as -2.270 , and defined ALBI grade $2 \mathrm{a}$ as a score of $<-2.270$, and $m A L B I$ grade $2 b$ as a score of $\geq-2.270$. Using this mALBI grade, Hiraoka et al. [5] showed that even the patient group with a Child-Pugh score of 5 included a mixture of patients with mALBI grades 1 (62\%), 2a (33\%), and $2 \mathrm{~b}(5 \%)$. In other words, even though all patients had 
Fig. 1. Both the Child-Pugh grade (a) and the mALBI (mALBI) (b) grade performed well for prognostic stratification of 85,636 patients with HCC diagnosed between 2014 and 2015. Specifically, patient numbers were distributed uniformly across mALBI grades $2 \mathrm{a}$ and $2 \mathrm{~b}$; these groups had a median OS of 71 months and 40 months, respectively, despite both being in the same ALBI 2 category. Child-Pugh grade (c) and mALBI grade (d) in HCC patients who underwent resection of the tumor. Stratification performance using the mALBI grade was better than that using the Child-Pugh grade. Patient numbers were distributed uniformly across mALBI grades $2 \mathrm{a}$ and $2 \mathrm{~b}$, and there was a clear difference in median OS: 90 months for 2 a versus 62 months for 2b. Child-Pugh grade (e) and mALBI grade (f) of HCC patients who underwent ablation. Both mALBI grade and Child-Pugh grade showed good stratification performance. Patient numbers were distributed uniformly across mALBI $2 \mathrm{a}$ and $2 \mathrm{~b}$, and there was a clear difference in median OS between the groups: 82 months for 2 a versus 61 months for $2 \mathrm{~b}$. OS, according to the Child-Pugh grade (g) and mALBI grade (h), of patients who underwent transarterial chemoembolization. Both mALBI grade and Child-Pugh grade performed well for stratification. Patient numbers were distributed uniformly across mALBI $2 \mathrm{a}$ and $2 b$, and there was a clear difference in median OS between groups: 44 months for $2 \mathrm{a}$ versus 31 months for $2 \mathrm{~b}$. OS, according to Child-Pugh grade (i) and mALBI grade (j), of HCC patients who underwent systemic therapy. Stratification performance of the mALBI grade was better than that of the Child-Pugh grade. Specifically, there was a good distribution of patient numbers across $m A L B I$ grades $2 \mathrm{a}$ and $2 \mathrm{~b}$, and a clear difference in median OS: 11 months for $2 \mathrm{a}$ versus 7 months for $2 b$. (Modified from ref. [8] with permission.)

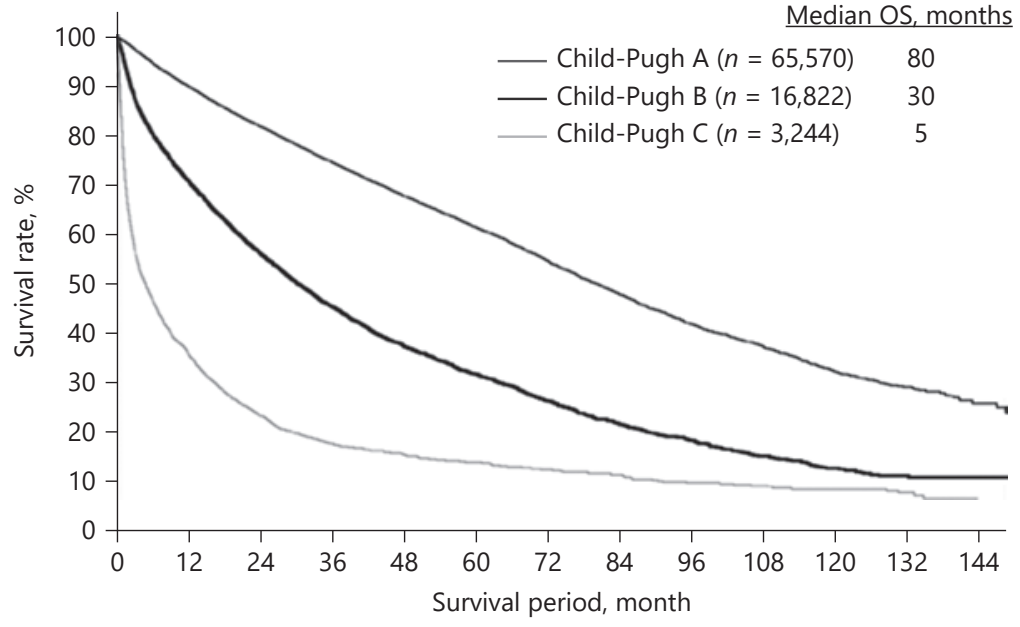

a

Hepatocellular carcinoma (all patients), stratified by Child-Pugh grade ( $n=85,636$ )

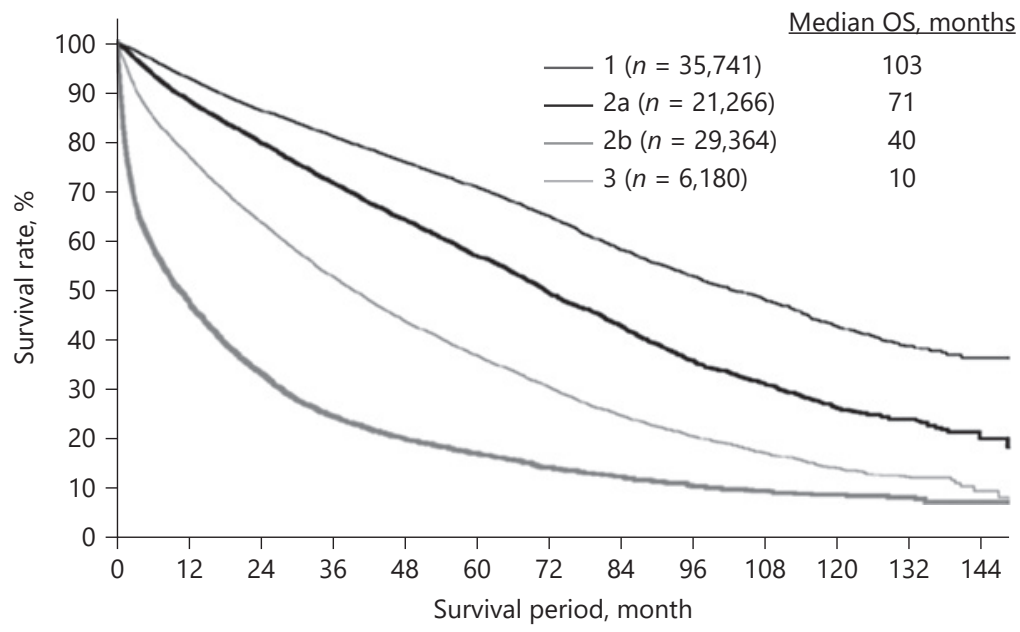

b

Hepatocellular carcinoma (all patients), stratified by mALBI grade $(n=92,551)$

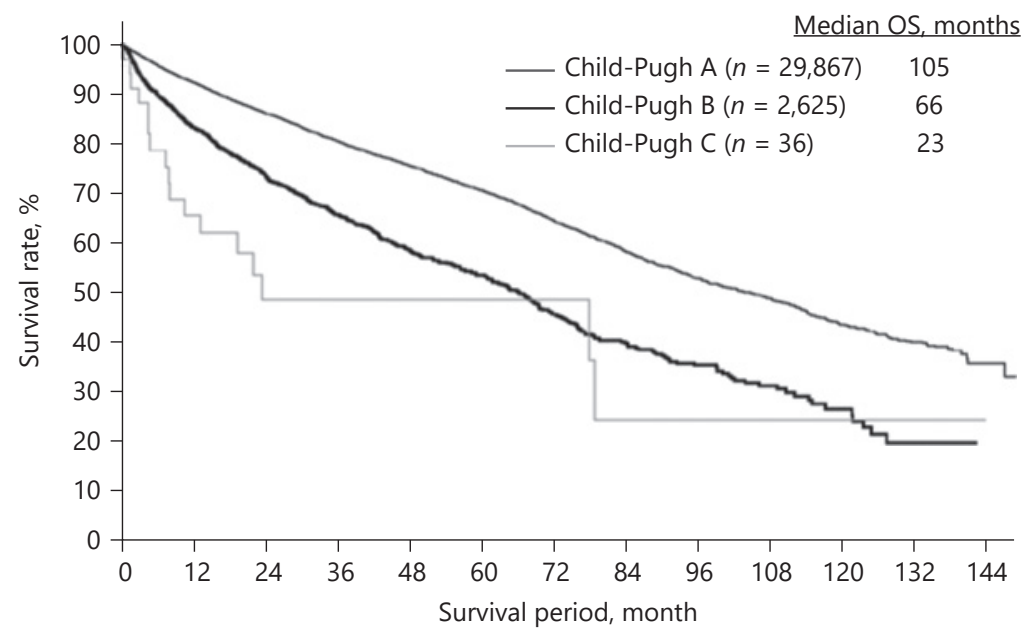

c Hepatocellular carcinoma (resection), stratified by Child-Pugh grade $(n=32,528)$

(Figure continued on next pages.) 


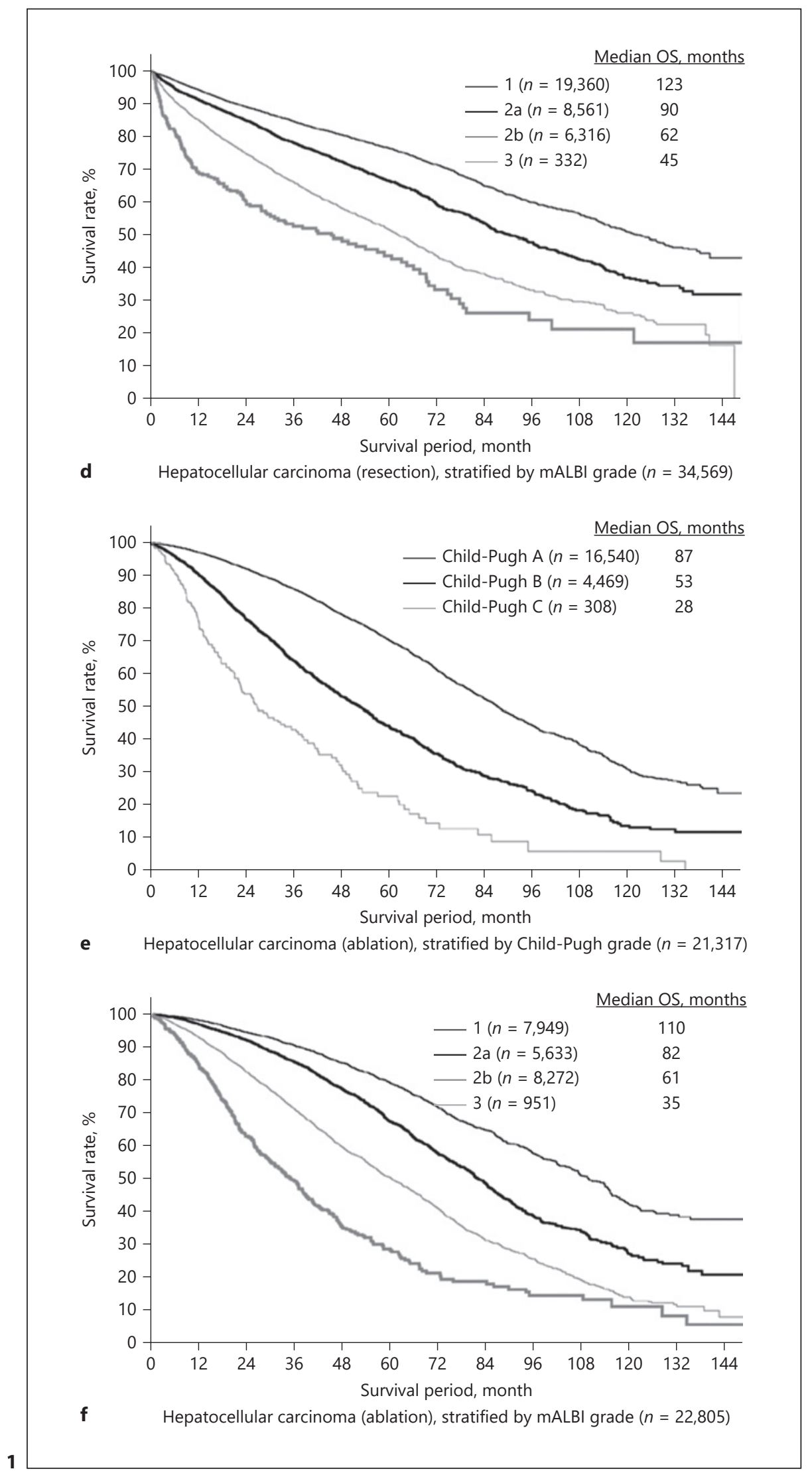

(Figure continued on next pages.) 


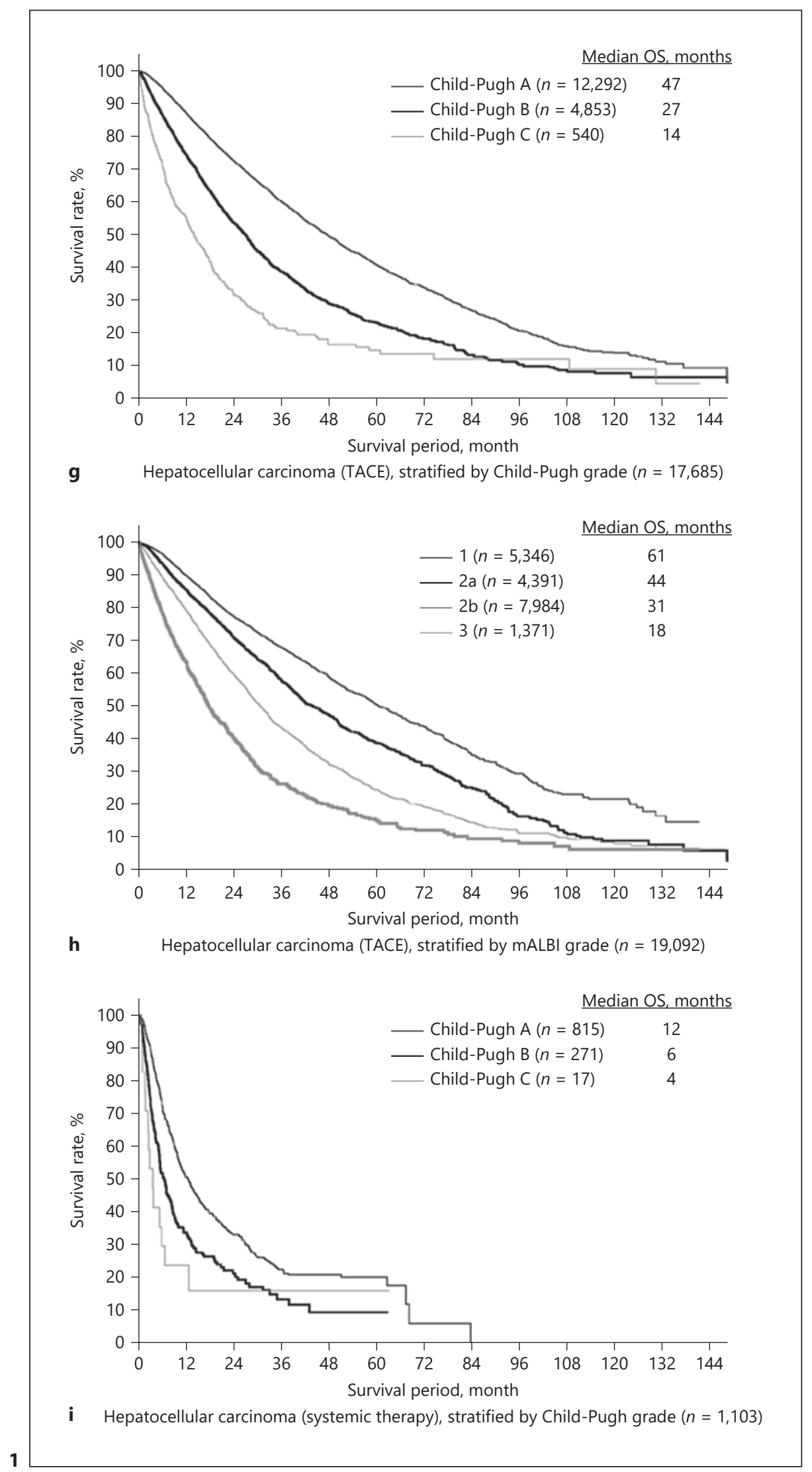

(Figure continued on next page.) 


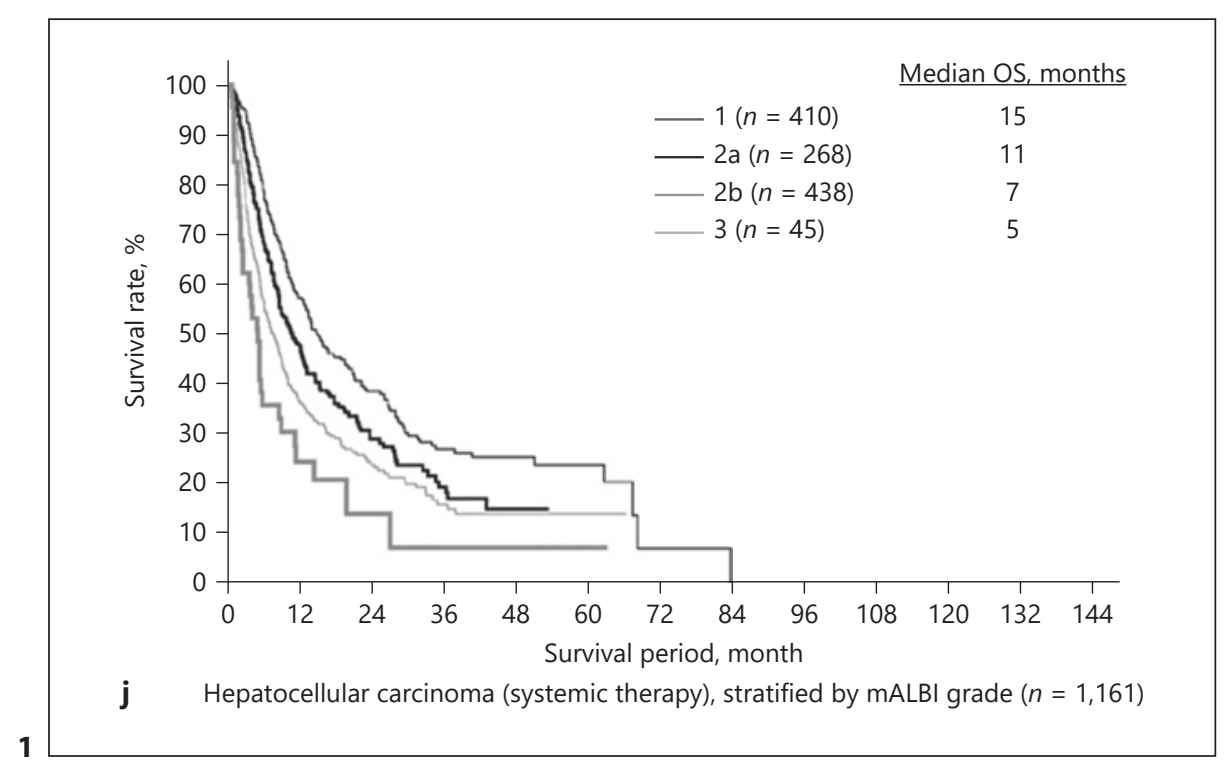

the same Child-Pugh score (i.e., 5), the group was extremely heterogeneous in terms of hepatic functional reserve. The group with a Child-Pugh score of 6 also had highly variable hepatic functional reserve: $11 \%$ were mALBI grade $1,23 \%$ were grade $2 \mathrm{a}$, and $66 \%$ were grade 2 b. Kumada et al. [7] reported similar results. These studies also demonstrate brilliantly that mALBI grades 1, 2a, $2 \mathrm{~b}$, and 3 are clearly stratified. In fact, looking at the data published to date, the report from the 23rd biannual survey of the Liver Cancer Study Group of Japan [8], as well as other recent studies $[5,9]$, show that the Child-Pugh grade was heavily skewed, with $72-85 \%$ of HCC patients graded as A; by contrast, the mALBI grade was well stratified, with $32-39 \%$ of patients graded as $1 ; 23-25 \%$ as 2 a; $32-40 \%$ as $2 \mathrm{~b}$; and $3-7 \%$ as 3 (Table 2). In particular, the miniscule difference in the proportions of mALBI grade $2 \mathrm{a}$ and $2 \mathrm{~b}$ patients is very important. In both Japan and the West, ALBI grade 3 patients have a very poor prognosis and are poor candidates for any treatment other than transplantation. Especially in Japan, the number of ALBI grade 3 HCC patients is very small, ranging from $3 \%$ to 7\% (Table 2). Thus, in practice, this makes the conventional ALBI grade essentially a 2-category system comprising ALBI grades 1 and 2. By contrast, the mALBI grade, which subdivides ALBI grade 2 into $2 \mathrm{a}$ and $2 \mathrm{~b}$, provides a balanced distribution of patients across grades 1 , $2 a$, and $2 b$, as well as showing excellent stratification performance between each score.

\section{Consistency of Prognostic and Predictive Value of mALBI Grade with Respect to Various Treatment Options}

Survey results from the Liver Cancer Study Group of Japan [8] showed that Child-Pugh grade (Fig. 1a) performed well with respect to stratification of $85,636 \mathrm{pa}$ tients with HCC; however, the 4-category mALBI grade system performed even better (Fig. 1b). The mALBI grade can also be used to identify patients with a more favorable prognosis; indeed, median overall survival (OS) for ChildPugh A patients is 80 months versus 103 months for mALBI grade 1 patients. In addition, the mALBI grade shows better performance than the Child-Pugh grade with respect to stratification of patients with resected HCC (Fig. 1c, d). The median OS at 123 months versus 105 months among patients with resected HCC was better for mALBI grade 1 patients than for Child-Pugh A patients. In addition, median OS at 45 months versus 23 months was better for mALBI grade 3 patients than for Child-Pugh C patients. For patients who underwent ablation therapy, mALBI grades $1,2 \mathrm{a}, 2 \mathrm{~b}$, and 3 also showed stratification performance comparable to that of ChildPugh grade (Fig. 1e, f). Median OS was better for mALBI grade 1 than for Child-Pugh A (110 months vs. 87 months, respectively), and for $m$ ALBI grade 3 than for Child-Pugh $\mathrm{C}$ (35 months vs. 28 months, respectively). The numbers of mALBI $2 \mathrm{a}$ and MALBI $2 \mathrm{~b}$ patients were distributed very uniformly, and the stratification performance of the mALBI grade was good for both the resection and ablation groups. In patients who underwent transarterial che- 
moembolization, the mALBI grade also showed very good stratification performance (similar to that of ChildPugh grade). It performed well both in terms of having a uniform distribution of grade 2 patients across grade $2 \mathrm{a}$ and $2 \mathrm{~b}$, and in terms of prognostic value (Fig. 1g, h). Median OS at 61 months versus 47 months was better for mALBI grade 1 than for Child-Pugh A. As observed in patients who underwent other treatments, median OS at 18 months versus 14 months was better for mALBI grade 3 than for Child-Pugh C. In patients who underwent systemic therapy, both the mALBI grade and the Child-Pugh grade showed very good stratification performance (Fig. 1i, j) [9-13]. In addition, median OS at 15 months versus 12 months was better for mALBI grade 1 than for Child-Pugh grade A, indicating that the mALBI grade is very useful as a prognostic factor. Furthermore, in patients who underwent systemic therapy, median OS was 11 months for mALBI grade 2a versus 7 months for mAL$\mathrm{BI}$ grade $2 \mathrm{~b}$, clearly indicating that there are subgroups with distinctly different prognoses even among ALBI grade 2 patients. Studies show that patients undergoing systemic therapy and who have a poor mALBI grade $(2 \mathrm{~b}$ or 3) do not respond as well to molecular targeted agents because they are more likely to experience adverse events and have dose intensity reduced as a result [9-14]. Consequently, it is very important to use the mALBI grade to identify which patients are in the mALBI $2 \mathrm{~b}$ subgroup [15].

\section{Conclusion}

Although the mALBI grade is used regularly in routine clinical practice in Japan, it is not yet used routinely worldwide. While the ALBI grade is certainly superior to the Child-Pugh grade, it has several drawbacks, mainly that ALBI grade 2 includes an oversized proportion of patients with a wide range of hepatic functional reserve, and that ALBI grade 3 includes very few patients. These are problematic because they effectively limit the ALBI system to just 2 categories: ALBI 1 and ALBI 2. Another problem is that ALBI 2 contains a mix of patients with a very good (mALBI $2 \mathrm{a}$ ) and poor (mALBI $2 \mathrm{~b}$ ) liver function and prognosis.

The mALBI grade proposed by Hiraoka et al. [5] results in a uniform distribution of patients across grades $2 \mathrm{a}$ and $2 \mathrm{~b}$. The results of a study based on data from a nationwide survey in Japan show that the mALBI grade is superior to the ALBI in that it produces a uniform distribution of patients among grades $2 \mathrm{a}$ and $2 \mathrm{~b}$ and improves stratification performance, indicating that the mALBI grade may be more useful than the ALBI grade for classifying hepatic functional reserve prior to any treatment. It is sincerely expected that this mALBI grade will become widely used not only in Japan, but also worldwide. With respect to clinical trials, we recommend use of the mALBI grade rather than the ALBI grade because the former enables more precise classification of hepatic functional reserve. However, it is also important in routine clinical practice to take advantage of the fact that the ALBI score is a continuous variable, and that, ultimately, the absolute ALBI score rather than the three-grade ALBI grade or the four-grade mALBI grade systems should be referenced when determining treatment strategy and predicting the prognosis of individual patients.

\section{Statement of Ethics}

Not applicable.

\section{Conflict of Interest Statement}

Lecture: Eisai, Bayer, MSD, BMS, EA Pharma, Eli Lilly, and Chugai; Grants: Eisai, Takeda, Otsuka, Taiho, EA Pharma, Gilead Sciences, Abbvie, Sumitomo Dainippon Pharma, Chugai, and Ono Pharma. Advisory Consulting: Eisai, Ono, MSD, BMS, and Roche. Masatoshi Kudo is the Editor-in-Chief of Liver Cancer.

\section{Funding Sources}

No funding was received.

\section{Author Contributions}

M. Kudo conceived, wrote, and approved the final manuscript.

\section{References}

1 Kudo M. Albumin-bilirubin grade and hepatocellular carcinoma treatment algorithm. Liver Cancer. 2017;6:185-8.

2 Johnson PJ, Berhane S, Kagebayashi C, Satomura S, Teng M, Reeves HL, et al. Assessment of liver function in patients with hepatocellular carcinoma: a new evidence-based approach-the ALBI grade. J Clin Oncol. 2015; 33:550-8.

3 Kariyama K, Nouso K, Hiraoka A, Wakuta A, Oonishi A, Kuzuya T, et al. EZ-ALBI score for predicting hepatocellular carcinoma prognosis. Liver Cancer. 2020;9(6):734-43. 
4 Hiraoka A, Kumada T, Kudo M, Hirooka M, Tsuji K, Itobayashi E, et al. Albumin-bilirubin (ALBI) grade as part of the evidence-based clinical practice guideline for HCC of the Japan Society of Hepatology: a comparison with the liver damage and Child-Pugh classifications. Liver Cancer. 2017;6:204-15.

5 Hiraoka A, Kumada T, Tsuji K, Takaguchi K, Itobayashi E, Kariyama K, et al. Validation of modified ALBI grade for more detailed assessment of hepatic function in hepatocellular carcinoma patients: a multicenter analysis. Liver Cancer. 2019;8:121-9.

6 Miyagawa S, Makuuchi M, Kawasaki S, Kakazu T. Criteria for safe hepatic resection. Am J Surg. 1995; 169:589-94.

7 Kumada T, Toyoda H, Tada T, Yasuda S, Tanaka J. Changes in background liver function in patients with hepatocellular carcinoma over 30 Years: comparison of Child-Pugh classification and albumin bilirubin grade. Liver Cancer. 2020;9:518-28.
8 Liver Cancer Study Group of Japan. Report of the 23th nationwide follow-up survey of primary liver cancer in Japan (2014-2015). Osaka; 2021. (in Japanese).

9 Tada T, Kumada T, Hiraoka A, Atsukawa M, Hirooka M, Tsuji K, et al. Impact of modified albumin-bilirubin grade on survival in patients with HCC who received lenvatinib. Sci Rep. 2021;11(1):14474.

10 Hiraoka A, Kumada T, Atsukawa M, Hirooka $\mathrm{M}$, Tsuji K, Ishikawa T, et al. Early relative change in hepatic function with lenvatinib for unresectable hepatocellular carcinoma. Oncology. 2019;97:334-40.

11 Hiraoka A, Kumada T, Tada T, Tani J, Kariyama K, Fukunishi S, et al. Efficacy of lenvatinib for unresectable hepatocellular carcinoma based on background liver disease etiology: multi-center retrospective study. Sci Rep. 2021;11(1): 16663 .
12 Tsuchiya K, Kurosaki M, Sakamoto A, Marusawa H, Kojima Y, Hasebe C, et al. The realworld data in Japanese patients with unresectable hepatocellular carcinoma treated with lenvatinib from a Nationwide Multicenter Study. Cancers. 2021;13(11):2608.

13 Ando Y, Kawaoka T, Suehiro Y, Yamaoka K, Kosaka Y, Uchikawa S, et al. Analysis of postprogression survival in patients with unresectable hepatocellular carcinoma treated with lenvatinib. Oncology. 2020;98:787-97.

14 Ueshima K, Nishida N, Hagiwara S, Aoki T, Minami T, Chishina $\mathrm{H}$, et al. Impact of baseline ALBI grade on the outcomes of hepatocellular carcinoma patients treated with lenvatinib: a Multicenter Study. Cancers. 2019; 11.

15 Kudo M, Kawamura Y, Hasegawa K, Tateishi R, Kariyama K, Shiina S, et al. Management of hepatocellular carcinoma in Japan: JSH consensus statements and recommendations 2021 Update. Liver Cancer. 2021;10(3):181223. 